\title{
Participación de los Municipios en la formación de la Política Nacional de Transporte Urbano en Venezuela*
}

\author{
Ocaña Ortiz, Rosa Virginia** \\ Urdaneta, Joheni ${ }^{\star \star \star}$
}

\section{Resumen}

El diseño e implantación de políticas públicas expresas en el área del transporte urbano en Venezuela data de finales de los años ochenta. En él se contempló la "modernización" y la "municipalización" del transporte urbano. El objetivo del presente trabajo es analizar la intervención de los poderes locales en el diseño e implementación de la política de transporte urbano en las ciudades venezolanas. La metodología utilizada consistió en el análisis de información documental y entrevistas semiestructuradas a funcionarios de Alcaldías y del gobierno central. Los resultados indican que la política fue diseñada en el seno del Ministerio de Transporte y Comunicaciones y del Ministerio de Infraestructura, por un grupo de especialistas, siguiendo lineamientos de la banca multilateral, sin consultar a los actores del sistema, y muy especialmente a los Municipios o Alcaldías. Se concluye que los poderes locales han intervenido de forma limitada en la implantación de la política, con muy escaso poder de decisión y siguiendo lo establecido por el gobierno nacional.

Palabras clave: Políticas públicas, transporte urbano, municipio, poderes locales.

\section{Recibido: 04-12-07. Aceptado: 05-05-05}

* Este trabajo forma parte de los resultados del Proyecto Políticas y Administración del transporte Urbano en las Alcaldías, adscrito al Programa de investigación Política y Administración Sub- Nacional del Centro de Estudios de la Empresa (CEE) de la Facultad de Ciencias Económicas y Sociales (FCES) de la Universidad del Zulia (LUZ). Financiado por el Consejo de Desarrollo Científico y Humanístico (CONDES) de LUZ.

** Profesora Titular del Departamento de Planificación Urbana, Universidad Simón Bolívar (USB). Valle de Sartenejas, Edif. MEU, Piso 1, Ofc. 114, Baruta, Edo. Miranda, CP 8900.

Tel (58-212) 906.40.94, Fax (58-212) 906.40.40. E-mail: rocana@usb.ve

*** Profesora Agregada de la Escuela de Ingeniería Industrial de la Facultad de Ingeniería e Investigadora del CEE de la FCES de LUZ. Estudiante del Doctorado en Ciencias Sociales, Mención Gerencia de LUZ. E-mail: joheniu@cantv.net 


\title{
Municipal Participation in the Formation of National Urban Transport Policy in Venezuela
}

\begin{abstract}
The design and implementation of expedient public policies in the area of urban transport in Venezuela began in the late 1980s. In this, the modernization and municipalization of urban transport is contemplated. The objective of this paper is to analyze the intervention of local leadership in the design and implementation of urban transport policy in Venezuelan cities. The methodology utilized was analysis of documentary information, and semi-structured interviews of municipal and national level officials. The results indicate that policies were designed in the Ministry of Transportation and Communication, and the Ministry of Infrastructure, by groups of specialists, following the guidelines of the multi-lateral bank, without consulting the actors within the system, and in particular local municipal officials. The conclusion is that local authorities have had a limited participation in the imposition of policy, have little decision making power, and have had to follow what has been established by the national government.
\end{abstract}

Key words: Public policy, urban transport, municipality, local power.

\section{Introducción}

El diseño e implantación de políticas públicas expresas en el área del transporte urbano en Venezuela data de finales de los años ochenta, con la Política Integral de Transporte Urbano (PITU) y, seguidamente, con las Políticas Nacionales de Transporte Urbano I y II (PNTU I y PNTU II).

Anteriormente, la mayoría de las acciones públicas en esa materia, correspondían a medidas aisladas, esporádicas, reactivas, a situaciones que se presentaban, mas no eran producto de una reflexión sobre los problemas y, mucho menos, del desarrollo de planes específicos en correspondencia con objetivos definidos.

En general, se tomaban medidas en áreas relacionadas al transporte, en concordancia con la situación económica del Estado venezolano (léase situación del petróleo). Estas medidas fueron distintas en materia de infraestructura y en materia del servicio de transporte público. La infraestructura fue asumida desde principios del siglo $X X$ mediante grandes inversiones en materia de vialidad.

En cuanto al servicio de transporte público, se presentaron en forma de mayor o menor regulación, distintos programas de subsidios a la operación y a la adquisición de vehículos (forma de redistribución de los ingresos petroleros), así como la aparición y liquidación de empresas públicas (concebidas como "modelos" a seguir por las organizaciones operadoras privadas).

Tanto la PITU como las PNTU contemplaron dentro de sus programas, medidas que planteaban elementos de "modernización" y de "municipalización" del transporte urbano. Modernización entendida como la creación de sólidas organizaciones de transporte urbano, que permitan superar la anarquía del servicio, la organización individual y las operadoras 
públicas ineficientes. Municipalización, entendida como la acción de permitir la toma de las decisiones por los actores locales, partiendo del principio que el transporte urbano responde a las realidades específicas de cada ciudad.

En este contexto, el rol del Estado sería propiciar y estimular la conformación de entes operadores privados o mixtos, que obedecerían a criterios modernos de administración y altos niveles de servicio, atendiendo a criterios sociales, de competitividad, de apertura de mercados, descentralización administrativa y crecimiento económico. Así, las diferentes políticas de transporte urbano diseñadas e implantadas por el nivel central se encuentran enmarcadas dentro del proceso de promoción de un nuevo modelo económico por parte del Estado y de un nuevo modelo de administración pública.

El tema de las políticas públicas en materia de transporte urbano ha sido poco estudiado en América Latina y aún menos en Venezuela ${ }^{1}$, donde sólo existen investigaciones parciales 0 indirectas. Particularmente, lo relativo a la participación de los gobiernos locales en el diseño e implantación de dichas y la capitalización de las experiencias de la gerencia pública con perspectiva científica en materia de servicios públicos se presentan como oportunidades de investigación.

Este trabajo presenta un análisis en el ámbito del transporte público urbano en Venezuela, el cual pudiera extrapo- larse a otros servicios y organizaciones públicas en América Latina.

De allí el interés de este trabajo, cuyo objetivo es analizar la intervención de los poderes locales en el diseño e implantación de la política de transporte urbano en las ciudades venezolanas. Se parte de la hipótesis de que la participación local en el diseño de la política es inexistente y la participación en la implantación es escasa.

El análisis realizado trabaja a profundidad la Política Nacional de Transporte Urbano I, diseñada en 1991 e implantada durante el período 1992-2000, y de manera parcial la Política Nacional de Transporte Urbano II, haciendo énfasis en su contenido.

\section{Sobre el Sistema de Transporte Urbano}

Se entiende por sistema de transporte urbano (STU) la conjunción de tres grandes subsistemas: transporte público, tránsito e infraestructura (vial y de transporte), pertenecientes a cualquier ámbito urbano. El subsistema de transporte público comprende lo relativo a la prestación del servicio (infraestructuras, vehículos, organizaciones operadoras, autoridades de tutela (Ministerios, Alcaldías), los usuarios (organizados o no), las relaciones entre ellos y el marco legal, entre otros.

El subsistema de tránsito involucra la operación del tránsito urbano y com-

1 Tal como ha sido expresado en reiteradas oportunidades por los expertos en transporte urbano en América Latina, en las conferencias de apertura y cierre de los Congresos Latinoamericanos de Transporte Público y Urbano. 
prende las vías, los sentidos de circulación, los equipos que regulan el tránsito, el cuerpo de vigilancia, la leyes de transito vigentes, entre otros. El subsistema vial se refiere a la construcción y mantenimiento de la infraestructura de transporte, entre las que destacan las vías (pavimento), las paradas de transporte colectivo, la señalización y demarcación.

El transporte de personas asegura principalmente la movilización de los trabajadores desde su lugar de residencia a los sitios de empleo, lo cual es masivo y condiciona gran parte de la actividad y la economía urbana. De esta manera, el transporte no es un fin en sí mismo, sino más bien un medio para mejorar las condiciones de vida en la ciudad. Así, el objetivo de una política de transporte no es simplemente movilizar a los usuarios, sino aumentar, de manera global, el bienestar de los habitantes y la productividad urbana.

Todo Sistema de Transporte Urbano cuenta con tres actores principales, los usuarios, los operadores y las autoridades de tutela. Cada uno de los tres actores juega un papel determinante e interactuante en el funcionamiento del sistema. Los intereses de cada uno son encontrados. En el caso específico del transporte público en Venezuela, todos los actores presentan una limitada capacidad financiera. Los usuarios poseen un bajo poder adquisitivo, las autoridades poseen presupuestos acotados, insuficientes para cubrir las necesidades que se presentan; $y$ los operadores de transporte presentan ingresos que escasamente cubren sus costos de operación por lo que no permiten realizar inversiones en sus unidades de transporte para mantener o mejorar la calidad del servicio.

Lo anterior demuestra la complejidad del problema del transporte urbano, y dado que este es un servicio público que cubre parte de las necesidades básicas de la población, el Estado debe tomar acciones para compensar y equilibrar las necesidades encontradas de los actores del sistema y crear las condiciones que permitan mejorar la calidad de vida de los ciudadanos. Estas acciones se denominan políticas públicas, según Ocaña (2003:16) "El término políticas públicas se refiere a un conjunto de acciones que toma alguna instancia gubernamental dirigidas a la resolución de situaciones que son percibidas como poco satisfactorias por la comunidad o por el ente que toma la medida, así como a mantener la calidad de vida de dicha comunidad".

En función de las necesidades expuestas, se han formulado en el país políticas nacionales de transporte urbano, con la finalidad de mejorar el servicio de transporte de las ciudades y, por ende, la calidad de vida de los ciudadanos.

\section{Políticas Nacionales de Transporte Urbano en Venezuela y Participación Municipal}

A los fines del presente trabajo, se considera el proceso de formación de la política tal como lo plantea Álvarez (1992), comprendido en dos fases, la de formulación, que comprende el establecimiento de objetivos y cursos de acción y la fase de implantación, a través de la cual se administra la política. 
Nos interesa la identificación de la participación de los gobiernos locales en cada una de las fases de las políticas mencionadas, para lo cual se utiliza la clasificación de la participación planteada por Cunill (1991), a saber: a) participación consultiva y/o asesora, expresada por opiniones y manifestaciones de conocimiento que no obligan al sujeto que adopta la decisión; b) participación resolutiva y participación fiscalizadora, que implican la intervención en el curso de la actividad pública y tiene una participación decisoria sobre alternativas relativas a la conducción de organizaciones o a la formulación de políticas, lo cual tiene un carácter obligante para la administración, y, c) participación en la ejecución, que consiste en tomar parte directamente en la realización de una actividad y/o en la prestación de un servicio.

Es importante resaltar que en Venezuela se han diseñado tres políticas expresas en materia de transporte: la Política Integral de Transporte (1989), la Política Nacional de Transporte Urbano I (1991) y la Política Nacional de Transporte Urbano II (2001).

\subsection{La política integral de transporte: ausencia del gobierno local}

La Política Integral de Transporte Público, fue diseñada durante el segundo mandato presidencial de Carlos Andrés Pérez (1989-1993). Este gobierno fue conocido por el establecimiento de una política neoliberal, que no sólo abarcó el ámbito económico, sino también el organizacional y el de los servicios urbanos, dentro de un modelo de administración publi- ca conocido como tecnocrático (Ochoa, 1995).

Según el MTC (1990), esta política surge como respuesta al creciente deterioro del servicio público. Aunque realmente fue producto de un conjunto de medidas económicas conocidas como el paquete que incluía la eliminación del régimen de cambios diferenciales y del control de cambios, liberación de las tasas de interés, ajuste en las tarifas de los servicios públicos y aumento de los precios de los derivados del petróleo, eliminando los subsidios. Estas medidas originaron un profundo malestar popular que se expresó en la explosión social ocurrida en Caracas y varias ciudades del país iniciadas por acciones de violencia colectiva en Guarenas, ciudad dormitorio de Caracas, motivada por el aumento desproporcionado del pasaje del transporte. Igualmente, "algunas vías centrales de las ciudades fueron tomadas por turbas, construyéndose barricadas y quemándose autobuses de transporte colectivo, vehículos privados y cauchos, en clara protesta contra los costos del servicio de transporte" (Fundación Polar, 1999:1).

Las acciones anteriormente expuestas obligaron al Estado a tratar de buscar una solución al problema del servicio de transporte urbano, por lo que se crea, de acuerdo al decreto presidencial del 20/04/1990, el Consejo Venezolano de Transporte (CVT), con la participación de cinco ministros, el presidente de la Compañía Metro de Caracas, el presidente del Instituto Autónomo de Ferrocarriles del Estado (FERROCAR), un representante de la Cámara de Industriales y Comercio (FEDECAMARAS), un repre- 
sentante de la Federación de Transporte en Venezuela (FEDETRANSPORTE) y el Gobierno Municipal representado por la Asociación Venezolana de Cooperación Intermunicipal (AVECl) (Urdaneta y Ochoa, 2004).

Es importante señalar que la participación decisoria de los municipios en el proceso de diseño de la política fue prácticamente nula, en el contexto de un Consejo integrado por 10 miembros y sólo un representante de los 300 municipios del país.

Este Consejo se creó con facultades decisorias con la finalidad de "definir los objetivos y prioridades del sector, revisar su marco institucional y regulatorio y hacer recomendaciones de reforma, evaluar políticas sectoriales y formular estrategias a corto y mediano plazo con la asistencia de agencias internacionales" (FONTUR, 2001), para ello se conformó un equipo de trabajo con expertos nacionales y brasileros, bajo la asesoría de los organismos multilaterales. Este equipo de expertos fue conformado por profesionales vinculados, en gran parte, al sector de consultoría, no hubo participación consultiva de los municipios, aun cuando éstos tenían la competencia y la experiencia en transporte público ya que para ese entonces esa responsabilidad era ejercida por el Concejo Municipal a través de diferentes figuras administrativas: direcciones de transporte, institutos de transporte, oficinas de transporte, o dentro de las responsabilidades de la oficina general de servicios públicos 0 de infraestructura.

Los lineamientos planteados para el diseño de la política tenían como fin "garantizarle al país un sistema de trans- porte urbano e interurbano eficiente, de buena calidad, en crecimiento y diversificación continua, y a un costo que genere los mayores beneficios a los usuarios y a los operadores" (MTC, 1990).

Esta política integral se enmarcó dentro de los Lineamientos Generales del VIII Plan de la Nación, partiendo del principio que "devuelve la eficiencia y competitividad a un sector tradicionalmente rentable pero largamente intervenido, protege a los grupos mas desasistidos a través de una asistencia directa a cada usuario y abre al público los beneficios de un servicio que crecerá y se diversificará, tanto en capacidad como en calidad y seguridad (MTC, 1990).

La Política Integral de Transporte Público tuvo su base sobre cuatro planes dirigidos a cada uno de los factores que determinan la operabilidad y viabilidad de los sistemas de transporte: a) Sistema dinámico de tarifas, este sistema consistía en "la asignación de tarifas diferenciales según la calidad del servicio prestado, el ajuste periódico de las tarifas, y la liberación de tarifas en aquellas rutas donde no existan razones sociales para que el gobierno intervenga" (MTC, 1990:5); b) Plan de subsidio social al transporte, proponía la compensación directa, a través de transferencias en efectivo, a los usuarios del transporte público, como desempleados, estudiantes, pensionados, jubilados y minusválidos; c) Plan de reducción de costos operativos, ofrecía diversas medidas entre las que destacan modificar la política arancelaria para importación de vehículos y repuestos; programa temporal (2 años) de subsidios para la adquisición de nuevos autobuses; exoneración de impuestos asociados a la adquisición 
de unidades de transporte público; y d) Reforma institucional del transporte, establecía tres tipos de acciones: i) Las reformas institucionales: revisión de leyes y regulaciones que impedían la libre operación de empresas organizadas en amplios sectores del transporte público; ii) los planes de inversión pública permitirían crear la infraestructura que facilitaría el desarrollo del transporte público y propiciarían la construcción de estacionamientos y terminales para las unidades de transporte periférico; $y$ iii) Los programas de apoyo al transporte público, planteaban el establecimiento de formas de vigilancia policial en las unidades de transporte periférico en las horas y rutas de mayor inseguridad; el estudio de las alternativas de incorporación al seguro social obligatorio de los operadores de transporte público y de los mecanismos de apertura y la promoción de inversionistas privados en sistemas modernos de transporte público (MTC, 1990).

Esta política, que perseguía "reorientar el transporte público hacia su recuperación definitiva y modernización permanente" (MTC, 1990:2), sólo fue parcialmente implementada y de manera exclusiva en el transporte interurbano cuya competencia es de los gobiernos Estadales (Nivel intermedio entre el nacional y el municipal), se circunscribió a la aplicación de tarifas diferenciales, según la calidad del servicio, para la cual existía una demanda latente, y la modificación de la política arancelaria para la importación de autobuses, que fue fácil de aplicar dada la inexistencia de empresas productoras de autobuses para servicio interurbano en el país.
En este sentido, la política no se implementó en lo referente al transporte urbano, a pesar de lo enunciado en los documentos y discursos originales, por lo que la participación de los Municipios en la ejecución de esta política fue completamente nula. Por otra parte, en el transporte interurbano tampoco se tomaron acciones públicas en materia de subsidio social al transporte, reducción de costos operativos o reforma institucional.

Como resultado de la implantación de tarifas diferenciales, se creó una gran variedad de tipos de servicio y de tarifas para viajes interurbanos en autobús, lo cual diversificó de manera importante la oferta. Paralelamente, proliferaron pequeños terminales de mejor calidad, diseño y comodidad que los existentes. Todos estos nuevos tipos de servicios de transporte interurbano continuaron desarrollándose a lo largo de la década de los años noventa y existen actualmente.

En el proceso de formación de la política integral de transporte urbano, los poderes municipales no participaron en ninguna de sus fases, ni en el diseño, ni en la implantación, a pesar de que había una pequeña representación de los Concejos Municipales (un representante de los 300 Municipios), en el organismo rector de esta política: el CVT. En el diseño, realizado por los expertos, no hubo participación consultiva de funcionarios municipales que trabajaban en el área de transporte urbano; y la implantación esta política no se llevó a cabo en el transporte urbano, cuya competencia es Municipal. 


\subsection{La Política Nacional de los noventa: participación de los municipios en la Implementación}

En el mismo contexto de la política anterior, se solicitó en 1991, un préstamo al Banco Mundial para la elaboración del proyecto "Transporte Urbano", a través del cual se desarrolló el Programa Nacional de Transporte Urbano, bajo las propuestas de este organismo (Urdaneta et al, 2002), y bajo la responsabilidad formal del MTC.

Al igual que en la Política Integral de Transporte Urbano, en la fase de formulación de la política participaron expertos nacionales ligados al sector de consultoría y los expertos brasileños como asesores internacionales pagados con el préstamo solicitado al Banco Mundial. No hubo participación de los poderes locales en esta fase.

La Política Nacional de Transporte Urbano I fue presentada mediante documento emanado del Ministerio de Transporte y Comunicaciones en 1991, atendiendo al "deterioro acumulado de los sistemas de transporte urbano, y conciente del nuevo papel que le corresponde asumir en el sector dentro del proceso de descentralización administrativa" (MTC, 1991:2). Se plantea que "este conjunto de políticas coherentes integrales, estables y definitivas sentará las bases para el mejoramiento gradual y permanente del transporte urbano, no solamente de los servicios que requiere con urgencia el país, sino también de la operación de la infraestructura vial en general" (MTC, 1991:2).

La política nacional de transporte urbano proponía cuatro grandes lineamientos: a) Municipalización de transpor- te urbano; b) Asistencia financiera del Estado; c) Creación de infraestructura de apoyo; y d) Modernización gerencial del transporte.

La municipalización del transporte parte del principio de que el transporte urbano responde a las realidades específicas de cada ciudad, por lo que al colocar las decisiones en los actores locales, se puede mejorar sustancialmente la calidad del servicio prestado. En el mismo orden de ideas, el documento de políticas planteaba que "los municipios son los principales responsables del transporte urbano, por lo que debían asumir en forma inmediata, permanente y prioritaria su obligación indelegable de garantizar (establecido en la Ley Orgánica de Régimen Municipal, -LORM- 1989) a la colectividad un transporte decente. Igualmente, debía crear una estructura organizativa definida técnicamente y dotada de los recursos humanos, materiales y financieros necesarios para el ejercicio de sus competencias, promoviendo operadores adecuados a los requerimientos y coordinación modal" (MTC, 1991:3).

Este lineamiento fue fundamental, de éste se derivaron los programas para la implementación de esta política y además estaba soportado con la reforma de ley de régimen municipal, donde se le transfería a los municipios todas las competencias del transporte urbano ${ }^{2}$, es decir, se le adicionaba la responsabilidad de la vialidad y el tránsito, que antes de la reforma era competencia exclusiva del MTC.

En cuanto, a la asistencia financiera del Estado, se plantea que éste debe instrumentar políticas de financiamiento integrales y dinámicas, capaces de gene- 
rar soluciones técnicas y operativas económicamente viables.

La creación de infraestructura de apoyo al transporte urbano plantea, a corto plazo, el respaldo del gobierno nacional a las alcaldías para que ejecuten mejoras físicas para el tránsito y la infraestructura de apoyo, con especial énfasis en la prioridad del transporte público. A largo plazo, se promovería el desarrollo de estudios y proyectos, para cada una de las ciudades, con miras a aumentar la eficiencia en la administración vial y la capacidad de transporte masivo, además de mejorar la estructura de las redes viales urbanas.

La modernización institucional parte de la necesidad de crear organizaciones sólidas de transporte urbano, que permitan superar la anarquía del servicio, la organización individual y las operadoras públicas ineficientes. En este sentido, correspondería a los municipios definir "el papel que deben prestar los servicios de transporte público en cada ciudad del país, con base en consideraciones técnicas, de complementariedad, eficiencia y eficacia en el nivel de servicio, las demandas y la economía, dentro del contexto de las condiciones peculiares de cada área urbana en particular, para lo cual formularán planes y proyectos que gradualmente mejoren las condiciones actuales y tiendan a la creación de sistemas locales de transporte urbano integrados" (MTC, 1991:4).

Los objetivos de la política nacional de transporte urbano serían: Eficiencia económica, cobertura de costos, justicia social, conveniencia administrativa y coordinación modal. Todo enmarcado dentro del Plan de la Nación denominado "EI Gran Viraje" y con tendencias neoliberales claras.

El programa estaría "inscrito en el proceso de descentralización administrativa y en el pleno reconocimiento de las competencias municipales en lo que se refiere al transporte y tránsito urbano"... mediante... "la asistencia técnica y financiera del FONTUR"... a los órganos locales (FONTUR, 2001:2). Para lograr este propósito se crearon tres subprogramas: a) Fortalecimiento Institucional, b) Mejoras físicas a la infraestructura y c) Renovación de flota de transporte público.

Para la ejecución de esta política, por recomendaciones del Banco Mundial, el Estado Venezolano creó la Fundación Fondo Nacional de Transporte Urbano (FONTUR), como organismo adscrito al Ministerio de Transporte y Comunicaciones. FONTUR fue creado bajo el concepto de un organismo altamente calificado desde el punto de vista técnico, con una cierta especificidad en materia de transporte urbano y dentro de la estructura del MTC, de allí que se adoptara la figura de Fundación y que la presidencia de la Junta Directiva correspondiese al Ministro. La figura permitiría el manejo de recursos externos (banca multilateral) y una administración menos complicada y burocrática que la existente en los ministerios, siguiendo las tesis dominantes de "modernización" del aparato público, las cuales plantearon la creación de institutos autó- 
nomos o semi-autónomos (CLAD, 1998) con la finalidad de lograr la eficiencia estatal, y contribuir a impulsar la economía de mercado (Urdaneta et al., 2002).

Antes de su creación, se vislumbró como una organización de corta vida, de seis a ocho años, que se proponía "sembrar" las semillas de la modernización del transporte urbano, cuyos frutos serían entre otros: pequeños fondos locales de transporte urbano; oficinas locales de transporte urbano en cada municipio con más de 100.000 habitantes; empresas privadas operadoras del transporte público urbano, en sustitución de las asociaciones civiles y cooperativas; un servicio de transporte público integrado y jerarquizado en cada ciudad, basado en un sistema troncal de autobuses de gran tamaño, alimentados por vehículos de pequeño porte en los ejes de menor importancia, con terminales de integración.

Este organismo, concentraría sus acciones en la implementación de los tres subprogramas: a) fortalecimiento institucional, b) mejoras a la infraestructura y c) financiamiento de flota, teniendo como fuente de recursos el presupuesto nacional y organismos de la banca multilateral. Su relación con las alcaldías se realizaría a través de la atención de las necesidades propias de cada municipio, expresadas en planes y proyectos, mediante financiamiento conjunto nacional y local.

Cada uno de estos subprogramas fue ejecutado a través de acciones diversas durante el período de estudio. A continuación se describe en que consiste cada subprograma y como fue la participación de los Municipios en cada una de ellas.

a) El subprograma de fortalecimiento institucional implicó el desarrollo de cursos de capacitación para transportistas y técnicos del transporte, la realización de seminarios, la asistencia técnica a oficinas municipales de transporte, el apoyo para la adquisición de mobiliario y equipos de oficinas (computadoras, impresoras, fax, fotocopiadoras, etc.), y la ejecución de estudios y proyectos de transporte y tránsito. Estas acciones se concibieron como elementos que permitían crear plataforma para apoyar la municipalización y ayudar a lograr la modernización gerencial (MTC, 1991). Las acciones para implantar este subprograma se decidieron en FONTUR, donde también se seleccionaron los Municipios a los cuales se les dotaría de equipos. Los Municipios seleccionados participaron enviando un requerimiento formal, a través de la presentación de los llamados Planes de fortalecimiento, con la descripción de las necesidades en materia de dotación de equipos de oficina. Por otra parte, el Fondo realizó una programación (unidireccional y centralizada) de cursos para técnicos (cuyo contenido no fue consultado a los Municipios), los ofreció a las alcaldías y les solicitó enviar a sus postulados. En el caso de cursos para transportistas, las alcaldías no realizaron ningún tipo de participación.

b) El subprograma de mejoras a la infraestructura implicó básicamente la contratación de proyectos de ingeniería de detalle, y la realización de obras (mantenimiento vial, demarcación, señalización, construcción de paradas, implantación de semáforos, construcción de pequeños terminales, etc.), incluyendo la inspección. Estas intervenciones creaban o mejoraban la infraestructura de transporte público. 
La participación de las alcaldías para la implementación de este programa fue de ejecutora, ya que el financiamiento se realizó mediante la presentación de planes y proyectos elaborados por las Alcaldías, o aquéllos desarrollados por FONTUR, aunque se debe señalar que los proyectos que realizan las Alcaldías tienen que estar enmarcados dentro de las propuestas de proyectos exigidos por los Organismos Multilaterales para otorgar el financiamiento (Urdaneta et al., 2001). En general, el personal de las Alcaldías puede emitir opiniones técnicas sobre los proyectos, aunque no siempre son consideradas o respetadas. Es necesario destacar que aunque se han realizados importantes gastos en las obras de construcción de proyectos, existen numerosos casos de inconformidad entre los representantes de alcaldías y vecinos, porque no cumplen las normas de construcción por ubicarse en zonas inadecuadas para equipamientos urbanos, presentan un diseño ineficiente (lo cual impide fluidez en la circulación interna), y una muy mala calidad en los materiales usados para su construcción (Ocaña, 2003, 118).

Para hacer uso de los recursos provenientes de la Banca Multilateral se solicita cumplir con una serie de etapas establecidas por esos organismos, así como con formatos de presentación de documentos, y proyectos. En resumen, son los organismos de la Banca los que aprueban los procesos, las contrataciones y supervisaban las obras (Urdaneta et al, 2002).

Asimismo, se evidencia que a pesar de las banderas del gobierno, de sustituir la administración pública por la ge- rencia pública (Ochoa, 1997: 128), buscando aligerar procesos y hacer más eficiente a los organismos públicos, los desembolsos de dinero para la ejecución de obras y proyectos realizados por los organismos descentralizados creados, presentan tiempos similares, y hasta mayores, a los de los ministerios.

c) El programa de renovación de flota se circunscribió a la adquisición de vehículos autobuses, minibuses y rústicos. En el documento de política se planteó de forma expresa que se privilegiarían los autobuses (por sus virtudes como medio de transporte colectivo, comparado con el minibús) y los rústicos (por servir las zonas donde vive la población menos favorecida económicamente). En Venezuela, históricamente se ha asumido la práctica de subsidiar la adquisición de vehículos de transporte público para evitar o frenar aumentos de tarifas de transporte. Asimismo, se ha creído que la modernización del transporte involucra necesariamente la compra de vehículos nuevos.

Además de las premisas explicadas anteriormente, dentro de las acciones para lograr la modernización del transporte, se manejó la tesis de apoyar la creación y desarrollo de empresas de transporte bajo la figura legal de derecho privado de compañías anónimas. En este sentido, se dio por hecho que al obligar a los transportistas a presentar entre los recaudos, para obtener créditos para la adquisición de vehículos nuevos (bajo el subprograma de renovación de flota), la documentación de una compañía anónima, se modificaría no sólo la razón social de la operadora, sino también su forma de funcionamiento, trayendo como con- 
secuencia la sustitución de las asociaciones civiles y cooperativas por dichas compañías y por tanto la modernización.

En este subprograma de renovación de flota la participación de las Alcaldías fue completamente nula, ya que el financiamiento a las operadoras de transporte se realizó sin siquiera informar a las Alcaldías de los otorgamientos de créditos a las operadoras de transporte, los cuales no respondieron ni a los resultados de estudios realizados por el municipio, ni a aquéllos realizados por el mismo FONTUR, y mucho menos a las demandas de las comunidades.

En líneas generales, se puede afirmar que no hubo participación de los Municipios en el diseño de la política nacional de transporte urbano de 1991. De hecho, durante las entrevistas realizadas a técnicos del área de transporte urbano, funcionarios de Alcaldías y de organismos del gobierno central, se recogieron impresiones tanto del papel del poder local en el diseño e implantación de las políticas como en la pertinencia o no de las acciones ejercidas.

En este sentido, los técnicos en transporte que laboran en el organismo del poder local coincidieron en autocalificarse como excluidos en el diseño de cualquiera de las políticas de transporte expuestas en los párrafos anteriores.

La exclusión del poder local en la implantación de la política es aun más grave, si se considera que no existen y nunca han existido representantes de las alcaldías en el directorio de FONTUR, mientras si hay representación de transportistas y empresarios de la "industria" del transporte.

Una ilustración de la situación se presenta en una carta abierta enviada por el alcalde del municipio Caroní para ese momento, Dr. Clemente Scotto a los ministros de Hacienda, Cordiplan, Energía y Minas, Transporte y Comunicaciones y al presidente de PDVSA ${ }^{3}$, fechada el 7 de septiembre de 1995 (Scotto, 1995). En ella se expresa que "aunque celebramos que se esté pensando en el fortalecimiento del transporte público como programa previo o complementario al aumento de la gasolina, no deja de preocuparnos el enfoque parcial y centralista"... Asimismo... "hemos dicho que un incremento del precio de la gasolina esta justificado si y solo si, ello involucra una alianza entre los municipios y PDVSA para fortalecer el transporte público". Y finalmente, ... "un verdadero sistema de transporte público ... requiere... del rol protagónico de los municipios en el diseño de sus soluciones locales y en el manejo de los recursos financieros (el programa con el Banco Mundial no tiene respuesta a este planteamiento)".

\subsection{La Política Nacional de Transporte Urbano II: participación de los técnicos nacionales en el diseño}

En el contexto de un nuevo gobierno nacional, elegido en el año 1998 y una nueva constitución nacional "....aprobada en 1999, donde se introducen disposiciones tendentes a asegurar la participación te. 
ciudadana y a favorecer la descentralización como política nacional que profundiza la democracia y debe crear mejores condiciones para la prestación eficaz y eficiente de los cometidos estatales" (FONTUR, 2001:2); se diseña una nueva política nacional de transporte urbano, denominada: Política Nacional de Transporte Urbano II (o PNTU 2001), la cual se concentró en la revisión y ajuste de los lineamientos estratégicos de la PNTU I, proponiendo: la organización de los poderes públicos; la asistencia financiera al sector; el aumento de la calidad del transporte urbano; la capacitación y modernización del sector y la adecuación de la infraestructura al transporte urbano.

La organización de los poderes públicos planteó la implantación de mecanismos de integración y de coordinación intergubernamental, así como también la definición precisa de las relaciones entre el sector público y las empresas operadoras del transporte público. Asimismo, planteó la consideración de las orientaciones en materia de ordenamiento territorial y planificación urbana, y las políticas económicas, industriales, energéticas, ambientales, y de servicios públicos (FONTUR, 2001).

La asistencia financiera al sector se orientó, según FONTUR (2001), hacia la elaboración de un conjunto de propuestas, estrategias y mecanismos para el financiamiento de la infraestructura y la operación del transporte público, así como la motivación a la participación privada en la inversión de capitales y la optimización de los subsidios.

El aumento de la calidad se planteó como mejorar la accesibilidad, estimular la recuperación de áreas urbanas, pro- mover un servicio de transporte público regular, seguro y con tarifas al alcance de los usuarios (FONTUR, 2001).

La capacitación y modernización del sector estaba orientado a incrementar el nivel de eficiencia de las operadoras de transporte, mediante la ejecución de programas de capacitación, asistencia técnica y entrenamiento a los operadores, así como a los distintos entes en los tres niveles de gobierno responsables de la supervisión y control del transporte (FONTUR, 2001).

Finalmente, la adecuación de la infraestructura al transporte urbano se propuso bajo un enfoque de actuación integral a través de la mejora en la operación, con prioridad para el transporte público.

La Política Nacional de Transporte Urbano II fue diseñada por un equipo de expertos venezolanos, coordinado por un consultor chileno, quienes se abocaron a constituir mesas de trabajo para cada una de las áreas del transporte urbano: legal, de información, económico-financiera, de calidad, institucional, entre otros. Fueron constituidas por personas representantes de diversos organismos involucrados en el transporte. Es de hacer notar que ninguna mesa de trabajo contó con la asistencia de personal técnico o político de las alcaldías, ni tampoco con usuarios, lo que es contradictorio con los lineamientos de la Constitución Nacional de la República Bolivariana de Venezuela, donde se fomenta la participación ciudadana como parte del proceso de cambio que impulsa el gobierno nacional.

Así mismo, es importante señalar que "el proceso de formulación de la Política Nacional de Transporte Urbano de 2001, se ha concentrado en la revisión y 
ajuste de los lineamientos estratégicos generados en 1991, definiendo objetivos para cada uno de éstos y formulando acciones y metas concretas para su instrumentación" (FONTUR, 2001:14). Como se ha expuesto en los puntos anteriores, la política de 1991 se diseñó bajo la asesoría de los Organismos Multilaterales en el marco de un gobierno con tendencia neoliberal y el gobierno electo en 1998, presenta un discurso adverso al neoliberalismo por lo que resulta contradictorio el diseño y la implantación de esa política por parte del gobierno nacional.

En la implantación de esta política la participación de los poderes locales sigue siendo escasa, y se presenta la misma situación de la política anterior: financiamiento de compra de unidades nuevas a transportistas, sin el conocimiento de los municipios, sin estudios que justifiquen la ruta para la demanda de la comunidad; otorgamiento de placas de taxis por parte del gobierno central sin la consulta a los municipios donde operan; las obras que se realizan son elaboradas por las alcaldías, y en algunos casos por el mismo FONTUR, pero finalmente aprobadas por éste para su financiamiento; la capacitación es diseñada por FONTUR y la participación de las alcaldías se limita a enviar las postulaciones de técnicos y profesionales para la formación en cursos organizados por FONTUR, sin considerar las necesidades que realmente requieren los funcionarios que laboran en estas instituciones.

\section{Conclusiones}

En Venezuela se han diseñado y ejecutado tres políticas en materia de transporte. La primera de ellas, la Política Integral de Transporte Urbano, fue diseñada en 1989 por el Consejo Venezolano de Transporte (CVT). La segunda, la Política Nacional de Transporte Urbano de 1991, fue formulada bajo la responsabilidad del Ministerio de Transporte y Comunicaciones y para su implementación creó el Fondo Nacional de Transporte Urbano (FONTUR). Estas dos políticas se realizaron con la asesoría de Organismos de la Banca Multilateral, en el contexto de un gobierno con tendencia neoliberal. Uno de sus lineamientos fundamentales fue la municipalización del transporte, que consistió en apoyar a los municipios en materia de transporte urbano ya que con la Nueva Ley Orgánica de Régimen Municipal en 1989, les transfirió completamente esta competencia (transporte público, vialidad y tránsito). La tercera política, denominada Política Nacional de Transporte Urbano 2001, fue una reformulación de la política del 91, ya que no presentó cambios sustanciales. Básicamente presentó cambios de forma, es decir, incorporó elementos que permiten realizar una evaluación bajo una metodología de evaluación de políticas públicas.

Lo anteriormente expuesto permite concluir que la política nacional de transporte urbano vigente continúa manteniendo preceptos del neoliberalismo en el contexto de un país cuyo discurso es contrario al modelo neoliberal.

Por otra parte, la participación de los gobiernos locales en la formulación e implantación de las políticas nacionales de transporte urbano ha sido escasa. En la política diseñada en 1989, la participación de los Municipios fue nula, tanto en el diseño (a pesar de que hubo 
una mínima participación decisoria a través de la representación de los Concejos Municipales en el organismo rector de esa política), como en la ejecución, ya que esta política no se implementó en el transporte urbano cuya competencia es municipal.

En la Política de 1991, hubo participación ejecutoria de los Municipios ya que las políticas que se diseñaron en el seno del MTC, bajo la asesoría de los multilaterales, fueron ejecutadas por los Municipios seleccionados por el gobierno central.

En cuanto a la participación en la política de 2001, las alcaldías participaron como ejecutoras de los mismos programas que se implementaron en la política de 1991; la participación en el diseño (realmente fue una reformulación de la política de 1991) fue nula a pesar de existir un discurso de democratización, en el cual, la participación, y especialmente la participación ciudadana, juega un rol fundamental para el desarrollo del modelo de país que quiere impulsar el gobierno nacional.

\section{Referencias Bibliográficas}

Alvarez D. Ángel E. (1992), Análisis de Políticas Públicas. Publicaciones del CLAD. Caracas.

CLAD (1998), Una Nueva Gestión Pública para América Latina. Ediciones CLAD. Caracas.

Cunill, Nuria (1991), Participación Ciudadana. Publicaciones del CLAD. Caracas

FONTUR (2001), Política Nacional de Transporte Urbano 2001, Boletín Reporte I\&T, Caracas, Venezuela.
Fundación Polar (1999), www.fpolar.com. 01-04-2005.

Ministerio de Transporte y Comunicaciones (MTC) (1990), Documento de Política Integral del Transporte. MTC, Caracas, Venezuela.

Ministerio de Transporte y Comunicaciones (MTC) (1991), Documento de Política de Transporte Urbano. MTC, Caracas, Venezuela.

Ocaña O., Rosa Virginia (2003), Análisis y Evaluación del Programa Nacional de Transporte Urbano en Venezuela, 1991-2000. Trabajo de Ascenso, USB, Caracas.

Ochoa Henríquez, Haydée (1995), Tecnocracia y Empresas Públicas en Venezuela. Editorial de La Universidad del Zulia (EDILUZ). Maracaibo. Venezuela.

Ochoa Henríquez, Haydée (1997), La reforma de la administración publica en Venezuela: proyectos y realidad, Gestión y Política Publica, Vol. VI, № 1, México, primer semestre 1997, pp. 125-149.

Scotto, Clemente, Carta abierta, El Universal, 10 de septiembre de 1995.

Urdaneta, Joheni y Ochoa Henríquez, Haydée (2004). Principales Actores en la Formación de la Política de Transporte Urbano de la Alcaldía de Maracaibo. Memorias II Congreso Internacional de Gerencia. Maracaibo

Urdaneta, Joheni; Ochoa Henríquez, Haydée y Molero, Estílita (2002), Política y Administración del Servicio de Transporte Urbano en la ciudad de Maracaibo: los cambios a partir de la reestructuración económica. En: Miradas. Año 1. Nro. 1. Revista Venezolana de Ciencias Sociales. Universidad Nacional Experimental de Guayana. Venezuela. 
una mínima participación decisoria a través de la representación de los Concejos Municipales en el organismo rector de esa política), como en la ejecución, ya que esta política no se implementó en el transporte urbano cuya competencia es municipal.

En la Política de 1991, hubo participación ejecutoria de los Municipios ya que las políticas que se diseñaron en el seno del MTC, bajo la asesoría de los multilaterales, fueron ejecutadas por los Municipios seleccionados por el gobierno central.

En cuanto a la participación en la política de 2001, las alcaldías participaron como ejecutoras de los mismos programas que se implementaron en la política de 1991; la participación en el diseño (realmente fue una reformulación de la política de 1991) fue nula a pesar de existir un discurso de democratización, en el cual, la participación, y especialmente la participación ciudadana, juega un rol fundamental para el desarrollo del modelo de país que quiere impulsar el gobierno nacional.

\section{Referencias Bibliográficas}

Alvarez D. Ángel E. (1992), Análisis de Políticas Públicas. Publicaciones del CLAD. Caracas.

CLAD (1998), Una Nueva Gestión Pública para América Latina. Ediciones CLAD. Caracas.

Cunill, Nuria (1991), Participación Ciudadana. Publicaciones del CLAD. Caracas

FONTUR (2001), Política Nacional de Transporte Urbano 2001, Boletín Reporte I\&T, Caracas, Venezuela.
Fundación Polar (1999), www.fpolar.com. 01-04-2005.

Ministerio de Transporte y Comunicaciones (MTC) (1990), Documento de Política Integral del Transporte. MTC, Caracas, Venezuela.

Ministerio de Transporte y Comunicaciones (MTC) (1991), Documento de Política de Transporte Urbano. MTC, Caracas, Venezuela.

Ocaña O., Rosa Virginia (2003), Análisis y Evaluación del Programa Nacional de Transporte Urbano en Venezuela, 1991-2000. Trabajo de Ascenso, USB, Caracas.

Ochoa Henríquez, Haydée (1995), Tecnocracia y Empresas Públicas en Venezuela. Editorial de La Universidad del Zulia (EDILUZ). Maracaibo. Venezuela.

Ochoa Henríquez, Haydée (1997), La reforma de la administración publica en Venezuela: proyectos y realidad, Gestión y Política Publica, Vol. VI, № 1, México, primer semestre 1997, pp. 125-149.

Scotto, Clemente, Carta abierta, El Universal, 10 de septiembre de 1995.

Urdaneta, Joheni y Ochoa Henríquez, Haydée (2004). Principales Actores en la Formación de la Política de Transporte Urbano de la Alcaldía de Maracaibo. Memorias II Congreso Internacional de Gerencia. Maracaibo

Urdaneta, Joheni; Ochoa Henríquez, Haydée y Molero, Estílita (2002), Política y Administración del Servicio de Transporte Urbano en la ciudad de Maracaibo: los cambios a partir de la reestructuración económica. En: Miradas. Año 1. Nro. 1. Revista Venezolana de Ciencias Sociales. Universidad Nacional Experimental de Guayana. Venezuela. 
una mínima participación decisoria a través de la representación de los Concejos Municipales en el organismo rector de esa política), como en la ejecución, ya que esta política no se implementó en el transporte urbano cuya competencia es municipal.

En la Política de 1991, hubo participación ejecutoria de los Municipios ya que las políticas que se diseñaron en el seno del MTC, bajo la asesoría de los multilaterales, fueron ejecutadas por los Municipios seleccionados por el gobierno central.

En cuanto a la participación en la política de 2001, las alcaldías participaron como ejecutoras de los mismos programas que se implementaron en la política de 1991; la participación en el diseño (realmente fue una reformulación de la política de 1991) fue nula a pesar de existir un discurso de democratización, en el cual, la participación, y especialmente la participación ciudadana, juega un rol fundamental para el desarrollo del modelo de país que quiere impulsar el gobierno nacional.

\section{Referencias Bibliográficas}

Alvarez D. Ángel E. (1992), Análisis de Políticas Públicas. Publicaciones del CLAD. Caracas.

CLAD (1998), Una Nueva Gestión Pública para América Latina. Ediciones CLAD. Caracas.

Cunill, Nuria (1991), Participación Ciudadana. Publicaciones del CLAD. Caracas

FONTUR (2001), Política Nacional de Transporte Urbano 2001, Boletín Reporte I\&T, Caracas, Venezuela.
Fundación Polar (1999), www.fpolar.com. 01-04-2005.

Ministerio de Transporte y Comunicaciones (MTC) (1990), Documento de Política Integral del Transporte. MTC, Caracas, Venezuela.

Ministerio de Transporte y Comunicaciones (MTC) (1991), Documento de Política de Transporte Urbano. MTC, Caracas, Venezuela.

Ocaña O., Rosa Virginia (2003), Análisis y Evaluación del Programa Nacional de Transporte Urbano en Venezuela, 1991-2000. Trabajo de Ascenso, USB, Caracas.

Ochoa Henríquez, Haydée (1995), Tecnocracia y Empresas Públicas en Venezuela. Editorial de La Universidad del Zulia (EDILUZ). Maracaibo. Venezuela.

Ochoa Henríquez, Haydée (1997), La reforma de la administración publica en Venezuela: proyectos y realidad, Gestión y Política Publica, Vol. VI, № 1, México, primer semestre 1997, pp. 125-149.

Scotto, Clemente, Carta abierta, El Universal, 10 de septiembre de 1995.

Urdaneta, Joheni y Ochoa Henríquez, Haydée (2004). Principales Actores en la Formación de la Política de Transporte Urbano de la Alcaldía de Maracaibo. Memorias II Congreso Internacional de Gerencia. Maracaibo

Urdaneta, Joheni; Ochoa Henríquez, Haydée y Molero, Estílita (2002), Política y Administración del Servicio de Transporte Urbano en la ciudad de Maracaibo: los cambios a partir de la reestructuración económica. En: Miradas. Año 1. Nro. 1. Revista Venezolana de Ciencias Sociales. Universidad Nacional Experimental de Guayana. Venezuela. 